\title{
Binge Eating Tendencies and Anger Coping: Investigating the Confound of Trait Neuroticism in a Non-Clinical Sample
}

\author{
Anne M. Connolly ${ }^{1}$, Elizabeth Rieger ${ }^{1 *}$ \\ and Ian Caterson ${ }^{2}$ \\ ${ }^{1}$ School of Psychology, University of Sydney, NSW, Australia \\ ${ }^{2}$ School of Molecular and Microbial Biosciences, University of Sydney, \\ NSW, Australia
}

Objective: Binge eating has been found to be associated with anger suppression. However, the anger suppression measure used in previous research is highly saturated with trait neuroticism. Furthermore, the dichotomised view of anger coping as either 'in' or 'out' has been questioned. The present study investigated the influence of trait neuroticism on the relationship between binge eating and anger suppression. In addition, a broader measure of anger coping was utilised.

Method: One hundred forty non-clinical women completed measures of binge eating, anger coping and trait neuroticism.

Results: Anger suppression, rumination and a lack of assertion (i.e. anger inexpressiveness) were significantly correlated with binge eating tendencies. However, hierarchical regression analyses showed that the anger inexpressiveness measures were not predictive of binge eating tendencies over and above trait neuroticism. Furthermore, the association between anger inexpressiveness and binge eating tendencies was differentially affected by the separate facets of neuroticism.

Discussion: Results suggest that the relationship between anger inexpressiveness and binge eating tendencies is partially accounted for by the impulsiveness and depressive affect facets of neuroticism. Previous studies are questioned in light of these findings and areas for further research are considered. Copyright $(C$ 2006 John Wiley \& Sons, Ltd and Eating Disorders Association.

Keywords: anger suppression; binge eating; trait neuroticism

\section{INTRODUCTION}

* Correspondence to: Dr Elizabeth Rieger, School of Psychology, University of Sydney, NSW 2006, Australia.

Tel: +61 29351 2788. Fax: +61290365223.

E-mail: lizr@psych.usyd.edu.au
Theoretical models have posited a central role for negative emotion in the aetiology and maintenance of binge eating (Fairburn, Cooper, \& Shafran, 2003; McManus \& Waller, 1995; Stice et al., 2001). Research indicates that one-third of women with 
binge eating problems eat in response to negative emotion (Stice et al.), most commonly, anger, anxiety and depression (Arnow, Kenardy, \& Agras, 1995). Binge eating is thought to regulate the emotional experience by reducing awareness of the emotion (Fairburn et al.; Stice et al.).

Emotion regulation plays an important role in everyday life and refers to the way in which individuals attempt to influence what emotions they experience, and how they experience and express these emotions (Gross, Richards, \& John, 2006). Expressive suppression of emotion is a commonly used regulation strategy involving the modification of behaviour while fully experiencing emotional arousal (Gross \& John, 2003). However, while behavioural modification is achieved, expressive suppression fails to provide subjective relief, thus the negative emotion lingers and remains unresolved (Gross \& John).

Anger is an important emotion requiring regulation in everyday life. Research has found that expressive suppression of anger (i.e. anger suppression) is elevated in women with bulimic pathology across the eating disorders of anorexia nervosa: binge/purge subtype, bulimia nervosa and binge eating disorder (Waller et al., 2003). Non-clinical bulimic tendencies are also associated with increased levels of anger suppression (Meyer et al., 2005; Milligan \& Waller, 2000; Milligan, Waller, \& Andrews, 2002). In terms of the specific symptoms associated with anger suppression the evidence is equivocal, with research showing a relationship between anger suppression and laxative use in clinical samples (Waller et al.), and anger suppression and binge eating in non-clinical samples (Milligan \& Waller). It is to the latter association that the present study is addressed.

Anger suppression is defined as the frequent experience of angry feelings, accompanied by physiological arousal, in the absence of behavioural expression (Boddeker \& Stemmler, 2000; Spielberger, 1996). While this definition appears to capture the construct of expressive suppression adequately, research studies by Martin and Watson (1997) and Martin et al. (1999) have questioned the validity of the scale commonly used to measure anger suppression (i.e., the Anger-In Scale; Spielberger). Using this scale, Martin et al. (1999) demonstrated a significant association between anger suppression and somatic complaints, however this relationship was not significant after trait neuroticism was controlled for. The authors argued that the empirical links observed between high anger suppression and negative health outcomes may be due to the measure being highly saturated with trait neuroticism, rather than anger suppression specifically.

In light of these concerns, it is not clear if anger suppression is related to eating pathology independent of trait neuroticism. Since individuals who binge eat generally score high on measures of trait neuroticism (Bulik, Sullivan, \& Kendler, 2002), it is important to investigate this possible confound further.

In addition to the possible construct validity problems with the Anger-In Scale, the dichotomised view of anger coping as either 'in' (suppression) or 'out' (expression) has been questioned (Linden et al., 2003). The use of an alternative and broader measure of anger coping may yield a clearer interpretation of the relationship between anger coping and binge eating. Linden et al. have developed a questionnaire (the Behavioural Anger Response Questionnaire (BARQ)) that assesses a broader range of coping mechanisms involved in the experience of anger. While the questionnaire does not have a specific anger suppression factor, the subscales of Rumination (i.e. thinking about and ruminating upon the anger-inducing event and feelings) and a lack of Assertion appear to tap into a similar construct (i.e. anger inexpressiveness). Gross and John (2003) suggest an association between the three anger factors in maintaining that a lack of assertion, combined with expressive suppression of emotion, contributes to a ruminative style of thinking, thus increasing the likelihood that the emotion will remain unresolved. According to this proposition, unresolved anger is left to linger, possibly requiring further regulation efforts (e.g. binge eating) in vulnerable individuals.

The first aim of the study was to investigate the relationship between binge eating and anger suppression using the Anger-In Scale (Spielberger, 1999), while taking into account the possible confound of trait neuroticism. It was hypothesised that anger suppression would be related to binge eating, but that the relationship would not be significant over and above trait neuroticism. The second aim was to explore the relationship between binge eating and a broader measure of anger coping (i.e. the BARQ; Linden et al., 2003). While the entire questionnaire was used in an attempt to explore the broader relationship between binge eating and anger coping, it was hypothesised that the specific subscales of Rumination and Assertion would be positively and negatively correlated with binge eating, respectively. In addition, given that the Neuroticism measure consists of several subscales (Angry Hostility, Anxiety, Self-Consciousness, 
Vulnerability, Impulsiveness and Depression), the third aim of the study was to explore the differential impact of the Neuroticism facets on the relationship between anger inexpressiveness and binge eating.

\section{METHOD}

\section{Participants}

The participants were 140 first-year female psychology students. Binge eating tendencies are common in young adult females and college students (Halmi, Falk, \& Schwartz, 1981; Schotte \& Stunkard, 1987). A non-clinical sample was chosen to minimise the comorbid psychopathology inherent in a clinical sample and to obtain a broader range of binge eating tendencies. A female sample was recruited due to the likelihood of gender differences regarding anger (e.g. anger suppression is associated with bulimic tendencies for females, whereas state anger is associated with bulmic tendencies for males; Meyer et al., 2005).

The mean age of participants was 19.5 years $(\mathrm{SD}=2.57$; range $=17.5-35.5$ years $)$. The mean body mass index (BMI: $\mathrm{kg} / \mathrm{m}^{2}$ ), calculated using self-report data, was $21.52(\mathrm{SD}=3.51$; range $=$ 15.57-36.79). Sixty-three participants (45\%) reported current dieting. The demographic variables demonstrated minimal impact on the predictor and outcome variables and thus were not included in any analyses.

\section{Measures}

\section{Binge Eating Scale (BES)}

The BES measures 16 characteristics describing feelings/cognitions and behaviours related to binge eating (Gormally, Black, Daston, \& Rardin, 1982). Each characteristic is comprised of three or four statements ranging from no binge eating concerns to severe binge eating concerns. Respondents choose the statement that best reflects their eating tendencies. Items are summed to form a total score (range $=0-46$ ). The BES has acceptable test-retest reliability $(r=0.87)$ and is moderately related to binge eating severity using 28-day food diaries ( $r=0.20-0.40$; Timmerman, 1999). Research suggests that the BES primarily focuses on the loss of control component of binge eating (Timmerman). While the BES is typically used in obese or clinical binge eating disorder populations, the questionnaire has also been used in normal-weight university samples (Crowther, Sanftner, Bonifazi, \&
Shepherd, 2001; Hansel \& Wittrock, 1997) to categorise participants into binge eating and nonbinge eating groups. The current study chose to use the total score to yield a continuous measure of binge eating tendencies.

\section{Eating Disorders Examination - Questionnaire $(E D E-Q)$}

The EDE-Q, derived from the Eating Disorders Examination (EDE) clinical interview, is a selfreport questionnaire assessing the frequency of key eating disorder symptoms based on the previous 28 days (Fairburn \& Beglin, 1994). The behavioural items of 'consuming an unusually large amount of food' and 'loss of control over eating during these episodes' were used to assess the presence of objective binge eating. There is moderate agreement between the EDE and the EDE-Q when assessing the presence of objective binge eating episodes (kappa $=0.47$; Mond, Hay, Rodgers, Owen, \& Beumont, 2004). While there is general agreement that the EDE-Q is inadequate for diagnostic purposes, the questionnaire is nonetheless useful as a screening instrument for research purposes (Mond et al.).

State Trait Anger Expression Inventory-2: Anger-In Scale (STAXI-2: Anger-In)

The STAXI-2 is a self-report questionnaire measuring the experience and expression of anger. The current study utilised the Anger-In Scale, consisting of eight items indexing how often anger is experienced but suppressed (e.g. 'I boil inside, but I don't show it'). Items are rated on a 4-point scale, ranging from 'almost never' to 'almost always'. Individuals who score high on anger suppression regularly experience intense anger that they behaviourally suppress, rather than overtly expressing it (Spielberger, 1999). Alpha coefficients for Anger-In range from 0.73 to 0.84 (Spielberger).

\section{Behavioural Anger Response Questionnaire (BARQ)}

The BARQ is a 37-item self-report questionnaire assessing six anger coping factors, for example, Assertion (i.e. the tendency to calmly reason with or talk to the person involved) and Rumination (i.e. the degree to which an individual continues to think about the anger-inducing event). Other factors include: Direct Anger-Out, Avoidance, Social Support-Seeking and Diffusion. Items are scored on a 5-point scale ranging from 'rarely' to 'very frequently'. A high score indicates a high frequency of use of the anger coping strategy. The authors report acceptable levels of reliability and validity, 
including factor stability and internal consistency (e.g. Rumination alpha coefficient $=0.75$; Assertion alpha coefficient $=0.77$; Linden et al., 2003).

\section{NEO Personality Inventory-Revised:}

Neuroticism (NEO PI-R: N)

The NEO PI-R Neuroticism domain scale is a 48-item self-report questionnaire measuring the general tendency to experience negative affect, vulnerability to experience psychological distress and impaired coping. Six facet scores and a total score are computed. Items are scored on a 5-point scale ranging from 'strongly disagree' to 'strongly agree'. A high score indicates a high level of trait neuroticism. The facets include Angry Hostility, Anxiety, Depression, Self-Consciousness, Impulsiveness and Vulnerability. The authors report acceptable levels of reliability and validity, including factor stability and alpha coefficients ranging between 0.69 (Impulsiveness) to 0.86 (Angry Hostility; Costa \& McCrae, 1992).

\section{Procedure}

Ethical approval was obtained from the Human Research Ethics Committee and the study was advertised as 'investigating the way women experience and respond to emotion'. Participants completed all questionnaires in groups of 10, with the order of administration counterbalanced across sessions, for which they received course credit.

\section{Data Analysis}

To take into account multiple testing, a significance level of $p<0.01$ was used. All data were checked for multivariate normality. Pearson correlations were used to assess the relationship between the anger coping measures and binge eating tendencies (as measured by the BES). Group differences in anger coping were examined using $t$-tests for those who reported objective binge eating episodes (as measured by the EDE-Q), compared to those who did not. Hierarchical regressions assessed the ability of significant anger coping scales to predict BES scores over and above trait neuroticism. The differential impact of the separate neuroticism facets on the relationship between the significant anger coping scales and binge eating tendencies were then assessed using a series of hierarchical regressions.

\section{RESULTS}

\section{Summary Statistics}

Summary statistics are presented in Table 1. Means for the Anger-In scale and BARQ are comparable to published norms (Linden et al., 2003; Spielberger, 1999). Means for the Neuroticism scale, however are elevated compared to published norms for collegeaged females (e.g. Costa \& McCrae, 1992: Total score $=99.8)$. Forty-two participants $(30 \%)$ reported the presence of one or more objective binge eating episodes over the previous 28 days. This is comparable to previous research using college students (Schotte \& Stunkard, 1987). As expected for a non-clinical sample, the mean score for the BES falls into the no/mild binge-eating category (Greenco, Marcus, \& Wing, 1995).

\section{Relationship Between Anger Suppression and Binge Eating}

As shown in Table 2, there was a positive and significant correlation between Anger-In scores

Table 1. Means and standard deviations (SD) for Anger-In, BARQ, Neuroticism and BES scales

\begin{tabular}{|c|c|c|c|c|c|}
\hline Measure & Mean & SD & Measure & Mean & SD \\
\hline STAXI-2 & & & NEO PI: Neuroticism & & \\
\hline Anger-In & 19.25 & 4.95 & Total score & 147.61 & 25.16 \\
\hline BARQ & & & Anxiety & 26.60 & 5.61 \\
\hline Direct Anger-Out & 16.25 & 5.09 & Anger/hostility & 22.84 & 5.40 \\
\hline Assertion & 16.46 & 4.83 & Depression & 25.32 & 6.07 \\
\hline Social Support-Seeking & 21.64 & 5.00 & Impulsiveness & 25.49 & 4.14 \\
\hline Rumination & 20.26 & 4.76 & Self-consciousness & 25.16 & 5.27 \\
\hline Avoidance & 16.61 & 4.35 & Vulnerability & 22.20 & 5.51 \\
\hline Diffusion & 14.49 & 4.81 & BES total score & 12.19 & 7.29 \\
\hline
\end{tabular}

STAXI-2; State Trait Anger Expression Inventory-2. BARQ; Behavioural Anger Response Questionnaire. NEO-PI Neuroticism; NEO Personality Inventory Neuroticism domain. BES; Binge Eating Scale. 
Table 2. Relationship between anger coping and the BES (Pearson correlation) and anger coping group differences in objective binge eating ( $t$-test)

\begin{tabular}{lrrrrr}
\hline & \multicolumn{2}{c}{ BES } & & \multicolumn{2}{c}{ OBE } \\
\cline { 2 - 3 } \cline { 5 - 6 } & \multicolumn{1}{c}{$r$} & $p$ & & $t$ & $p$ \\
\hline STAXI-2 & & & & \\
$\quad$ Anger-In & 0.314 & 0.0005 & & -1.363 & 0.175 \\
BARQ & & & & \\
$\quad$ Assertion & -0.243 & 0.004 & & -0.311 & 0.756 \\
$\quad$ Direct Anger-Out & 0.159 & 0.060 & & -1.716 & 0.088 \\
$\quad$ Social support & -0.021 & 0.801 & & -0.129 & 0.897 \\
$\quad$ Rumination & 0.232 & 0.006 & & -1.347 & 0.180 \\
$\quad$ Avoidance & 0.103 & 0.225 & & -0.806 & 0.422 \\
$\quad$ Diffusion & -0.044 & 0.602 & & -0.661 & 0.510 \\
\hline
\end{tabular}

Significance level $=p<0.01$. STAXI-2; State Trait Anger Expression Inventory-2. BARQ; Behavioural Anger Response Questionnaire. BES; Binge Eating Scale. OBE; Objective binge eating episode (consuming a large amount of food with associated loss of control over eating).

on the STAXI-2 and BES scores. The group who reported objective binge eating (as indexed by the EDE-Q) did not differ in Anger-In scores, compared to the group who reported no objective binge eating.

While Anger-In was significantly associated with binge eating tendencies as measured by the BES, this relationship was not significant over and above Neuroticism $\left(\beta=0.155 ; d f=1,137 ; R^{2}\right.$ Change $=0.017$; $p=0.096)$.

\section{Relationship Between the BARQ and Binge Eating}

As shown in Table 2, there was a positive and significant correlation between Rumination scores and BES scores; and a negative and significant correlation between Assertion scores and BES scores. The group who reported objective binge eating (as indexed by the EDE-Q) did not differ in Rumination or Assertion scores, compared to the group who reported no objective binge eating.

Consistent with Anger-In results reported above, Rumination and a lack of Assertion were not significant over and above Neuroticism (Rumination: $\beta=0.029 ; d f=1,137 ; R^{2}$ Change $=0.001$; $p=0.759$ / Assertion: $\beta=-0.131 ; d f=1,137 ; R^{2}$ Change $=$ $0.015 ; p=0.115)$.

\section{Assessing the Differential Impact of Trait Neuroticism}

As shown in Table 3, the various facets of trait neuroticism demonstrated a differential impact on the relationship between anger inexpressiveness (Anger-In; Rumination; lack of Assertion) and the BES. Specifically, a series of hierarchical regression analyses controlling for the separate Neuroticism facets, showed that Self-Consciousness, Depression and Impulsiveness scores removed the largest

Table 3. Beta coefficients ( $p$-value) and unique variance ( $R^{2} \%$ Change) accounted for by Anger-In (STAXI-2), Rumination (BARQ) and Assertion (BARQ) scores in BES scores, controlling for Neuroticism facet scores

\begin{tabular}{|c|c|c|c|c|c|c|c|}
\hline & & \multicolumn{6}{|c|}{ Neuroticism Facets } \\
\hline & & Anxiety & Ang/Hos & Vulner & SelfCons & Depress & Impul \\
\hline $\begin{array}{l}\text { BES } \\
R^{2} \% \text { Change }\end{array}$ & $\begin{array}{c}\text { Anger-In }^{\dagger} \\
0.314(<0.0005) \\
9.9\end{array}$ & $\begin{array}{c}0.309(0.001) \\
8.2\end{array}$ & $\begin{array}{c}0.293(0.001) \\
7.4\end{array}$ & $\begin{array}{c}0.274(0.002) \\
6.3\end{array}$ & $\begin{array}{c}0.182(0.045) \\
2.5\end{array}$ & $\begin{array}{c}0.154(0.092) \\
1.7\end{array}$ & $\begin{array}{c}0.122(0.088) \\
1.3\end{array}$ \\
\hline \multirow{2}{*}{$R^{2} \%$ Change } & & \multicolumn{6}{|c|}{ Neuroticism Facets } \\
\hline & & Anxiety & Ang/Hos & Vulner & SelfCons & Impul & Depress \\
\hline BES & $\begin{array}{l}\text { Rumination }^{\dagger} \\
0.232(0.006)\end{array}$ & $0.220(0.021)$ & $0.198(0.038)$ & $0.177(0.051)$ & $0.094(0.287)$ & $0.073(0.295)$ & $0.041(0.657)$ \\
\hline \multirow[t]{3}{*}{$R^{2} \%$ Change } & 5.4 & 3.7 & 3.0 & 2.6 & 0.7 & 0.5 & 0.1 \\
\hline & & \multicolumn{6}{|c|}{ Neuroticism Facets } \\
\hline & & Anxiety & Ang/Hos & Vulner & SelfCons & Depress & Impul \\
\hline & Assertion $^{\dagger}$ & & & & & & \\
\hline BES & $-0.243(0.004)$ & $-0.225(0.009)$ & $-0.213(0.014)$ & $-0.204(0.017)$ & $-0.148(0.074)$ & $-0.143(0.080)$ & $-0.109(0.117)$ \\
\hline$R^{2} \%$ Change & 5.9 & 4.8 & 4.2 & 3.9 & 2.0 & 1.9 & 1.1 \\
\hline
\end{tabular}

Significance level $=p<0.01$. Anxiety; Anxiety. Ang/Hos; Angry Hostility. Vulner; Vulnerability. Impul; Impulsivity. SelfCons; Self-Consciousness. Depress; Depression. BES; Binge Eating Scale.

${ }^{\dagger}$ Bivariate beta coefficient. 
amount of variance in the relationship between anger inexpressiveness and the BES. For example, when using Anger-In as the predictor variable, 7.4, 8.2 and $8.6 \%$ of the variance in predicting BES scores were removed, when controlling for Self-Consciousness, Depression and Impulsiveness scores, respectively. Anger-In, however, still predicted BES scores over and above Anxiety, Angry Hostility and Vulnerability. There was a similar pattern of decreased unique variance accounted for by Rumination and a lack of Assertion, with Impulsiveness, Depression and Self-Consciousness scores removing the largest amount of variance in predicting BES scores.

\section{DISCUSSION}

Previous research has reported a relationship between anger suppression and bulimic pathology in clinical and non-clinical samples (Meyer et al., 2005; Milligan \& Waller, 2000; Milligan et al., 2002; Waller et al., 2003). The present study, however, suggests that the relationship between anger suppression and binge eating tendencies is partly attributable to the trait neuroticism facets of SelfConsciousness, Impulsivity and Depression.

The first aim of the study was to replicate previous research demonstrating a relationship between anger suppression and binge eating, and to investigate this relationship over and above trait neuroticism. The initial results of the current study partially replicated previous research. That is, bingeeating tendencies, as indexed by the BES, were related to higher Anger-In scores on the STAXI-2. Contrary to expectations, there were no group differences in anger suppression for those who reported objective binge eating, compared to those who did not. This finding is seemingly in contrast with research by Milligan and Waller (2000) who reported a significant association between the presence of anger suppression and binge eating using the Bulimic Investigatory Test Edinburgh (BITE). The BITE, however does not include questions on loss of control over eating and therefore provides a questionable index of objective binge eating. However, measurement problems may have also hindered the current study. The accuracy of self-report questionnaires to assess binge eating has been questioned (Wilfley, Schwartz, Spurrell, \& Fairburn, 1997). Research assessing the reliability of the EDE-Q, compared to the clinical interview, is equivocal. Studies have demonstrated an underestimation (Mond et al.,
2004; Wilfley et al.), and an overestimation (Fairburn \& Beglin, 1994) of the frequency of objective binge eating episodes. As such, future research should replicate the present study assessing binge eating status using a clinical interview rather than questionnaire data.

While partial agreement with existing literature was demonstrated in terms of a relationship between anger suppression and binge eating tendencies, previous studies have failed to take into account research suggesting that the Anger-In scale of the STAXI-2 is confounded with trait neuroticism (Martin \& Watson, 1997; Martin et al., 1999). Supporting results obtained by Martin et al., the present study found that when Neuroticism was controlled for, Anger-In was no longer predictive of BES scores. This finding, thus questions previous research in the eating disorders literature, suggesting that the relationship between anger suppression and binge eating is confounded by neuroticism (at least utilising the Anger-In scale).

To determine whether the confounding role of neuroticism is specific to the Anger-In scale, an alternative and broader measure of anger coping (BARQ) was utilised in relation to binge eating. Results demonstrated that rumination and a lack of assertion were related to binge eating tendencies as indexed by the BES. However, once again these relationships were not significant over and above trait neuroticism. Thus, the use of an alternative anger coping measure also showed that neuroticism confounded the relationship between anger coping and binge eating tendencies.

Neuroticism, however, consists of several facets. Analyses controlling for the separate facets showed a distinct pattern of differential impact on the primary relationship, with Self-Consciousness, Depression and Impulsiveness scores removing the largest amount of variance in the association between Anger-In and BES scores. Anger-In, however, was still predictive of BES scores, over and above Anxiety, Angry Hostility and Vulnerability. Rumination and a lack of Assertion showed a similar pattern to Anger-In. Therefore, the relationship between anger inexpressiveness and binge eating tendencies seems to be attributable to the individual's predisposition to be impulsive, experience self-consciousness and depressive affect specifically, rather than neuroticism generally as the research of Martin et al. (1999) suggests.

Given that the BES measures impulsive eating, the confounding influence of Impulsiveness is perhaps not surprising. However, an explanation for the confounding influence of depression is less 
self-evident. One possibility is that the anger inexpressiveness measures have construct validity problems in terms of partially measuring depressive affect rather than constituting pure measures of anger inexpressiveness. For example, the Anger-In item 'I withdraw from people' is more consistent with depressive symptomatology; whereas the item 'I boil inside, but I don't show it' seems to more clearly index anger suppression. Similarly, some items on the Rumination scale may index depressive affect (e.g. 'In my mind, I try to figure out why I really got upset'); while other items are more suggestive of anger rumination (e.g. 'I develop a revenge fantasy where I "get even" with the person who angered $\mathrm{me}^{\prime}$ ).

Alternatively, the current findings may be due to a genuine relationship between anger inexpressiveness and depressive affect, rather than arising from deficiencies in the measures employed to assess anger inexpressiveness. For instance, prospective research has shown that anger suppression predicts depression (Bromberger \& Matthews, 1996). Depression is also related to anger-focused rumination (Gilbert, Cheung, Irons, \& McEwan, 2005), and to the fear of anger (Brody, Haaga, Kirk, \& Soloman, 1999). Assertiveness training is also effective in decreasing depression and binge eating episodes in women with binge eating disorder (Clyne \& Blampied, 2004). These findings suggest that anger inexpressiveness may act as a vulnerability factor for depressive symptomatology. Feelings of inauthenticity and internal conflict generated by expressive suppression (Gross et al., 2006) and a ruminative style of coping may decrease the likelihood of emotional resolution, thus contributing to and maintaining depressive affect (NolenHoeksema, 1991). A similar mechanism may also account for the confounding influence of SelfConsciousness in the relationship between binge eating and anger suppression, given that the Neuroticism facet of Self-Consciousness measures a sense of worthlessness (e.g., 'I often feel inferior to others.'). Vulnerable individuals may, therefore need to further regulate their emotions by binge eating. This hypothesis, however requires experimental research in order to establish the direction of effect and the exact role of anger in this equation. Replication of the results in a clinical sample is also recommended. In addition, due to the elevated levels of trait neuroticism in the current study, compared to normative data (Costa \& McCrae, 1992), replication of the results is suggested.

Overall, the current study provides initial evidence questioning the validity of the relationship between anger suppression and bulimic tendencies in women, previously reported in the literature. Results demonstrated that the relationship between anger inexpressiveness and binge eating tendencies was no longer significant after controlling for impulsiveness and depressive affect. Further research is needed to determine if the anger inexpressiveness scales used in the present study are plagued by construct validity problems, or whether the association between anger inexpressiveness and binge eating is genuine, being partially accounted for by self-consciousness, impulsiveness and depressive affect.

\section{ACKNOWLEDGEMENT}

Anne M. Connolly thanks and acknowledges the New South Wales Institute of Psychiatry for a Research Training Fellowship.

\section{REFERENCES}

Arnow, B., Kenardy, J., \& Agras, W. S. (1992). Binge eating among the obese: A descriptive study. Journal of Behavioral Medicine, 15, 155-170.

Arnow, B., Kenardy, J., \& Agras, W. S. (1995). The Emotional Eating Scale: The development of a measure to assess coping with negative affect by eating. International Journal of Eating Disorders, 18, 79-90.

Boddeker, I., \& Stemmler, G. (2000). Who responds how and when to anger? The assessment of actual anger response styles and their relation to personality. Cognition and Emotion, 14, 737-762.

Brody, C. L., Haaga, D. A. F., Kirk, L., \& Solomon, A. (1999). Experiences of anger in people who have recovered from depression and never-depressed people. Journal of Nervous and Mental Disease, 187, 400-405.

Bromberger, J. T., \& Matthews, K. A. (1996). A 'feminine' model of vulnerability to depressive symptoms: A longitudinal investigation of middle-aged women. Journal of Personality and Social Psychology, 70, 591-598.

Bulik, C. M., Sullivan, P. F., \& Kendler, K. S. (2002). Medical and psychiatric morbidity in obese women with and without binge eating. International Journal of Eating Disorders, 32, 72-78.

Clyne, C., \& Blampied, N. M. (2004). Training in emotion regulation as a treatment for binge eating: A preliminary study. Behaviour Change, 21, 269-281.

Costa, P. T., Jr., \& McCrae, R. R. (1992). Revised NEO personality inventory (NEO PI-R) and NEO five-factor inventory (NEO-FFI) professional manual. Odessa, Florida: Psychological Assessment Resources, Inc.

Crowther, J. H., Sanftner, J., Bonifazi, D. Z., \& Shepherd, K. L. (2001). The role of daily hassles in binge eating. International Journal of Eating Disorders, 29, 449-454. 
Fairburn, C. G., \& Beglin, S. J. (1994). Assessment of eating disorders: Interview or self-report questionnaire? International Journal of Eating Disorders, 16, 363-370.

Fairburn, C. G., Cooper, Z., \& Shafran, R. (2003). Cognitive behaviour therapy for eating disorders: A "transdiagnostic" theory and treatment. Behaviour Research and Therapy, 41, 509-528.

Gilbert, P., Cheung, M., Irons, C., \& McEwan, K. (2005). An exploration into depression-focused and angerfocused rumination in relation to depression in a student population. Behavioural and Cognitive Psychotherapy, 33, 273-283.

Gormally, J., Black, S., Daston, S., \& Rardin, D. (1982). The assessment of binge eating severity among obese persons. Addictive Behaviors, 7, 47-55.

Greenco, C. G., Marcus, M. D., \& Wing, R. R. (1995). Diagnosis of binge eating disorder: Discrepancies between a questionnaire and clinical interview. International Journal of Eating Disorders, 17, 153-160.

Gross, J. J., \& John, O. P. (2003). Individual differences in two emotion regulation processes: Implications for affect, relationships, and well-being. Journal of Personality and Social Psychology, 85, 348-362.

Gross, J. J., Richards, J. M., \& John, O. P. (2006). Emotion regulation in everyday life. In D. K. , Snyder, J. A. , Simpson, \& J. N. Hughes (Eds.), Emotion regulation in families: Pathways to dysfunction and health. Washington D.C.: American Psychological Association.

Halmi, K. A., Falk, J. R., \& Schwartz, E. (1981). Binge-eating and vomiting: A survey of a college population. Psychological Medicine, 11, 697-706.

Hansel, S. L., \& Wittrock, D. A. (1997). Appraisal and coping strategies in stressful situations: A comparison of individuals who binge eat and controls. International Journal of Eating Disorders, 21, 89-93.

Linden, W., Hogan, B. E., Rutledge, T., Chawla, A., Lenz, J. W., \& Leung, D. (2003). There is more to anger coping than 'in' or 'out'. Emotion, 3, 12-29.

Martin, R., \& Watson, D. (1997). Style of anger expression and its relation to daily experience. Personality and Social Psychology Bulletin, 23, 285-294.

Martin, R., Wan, C. K., David, J. P., Wegner, E. L., Olson, B. D., \& Watson, D. (1999). Style of anger expression: Relation to expressivity, personality, and health. Personality and Social Psychology Bulletin, 25, 11961207.
McManus, R., \& Waller, G. (1995). A functional analysis of binge-eating. Clinical Psychology Review, 15, 845-863.

Meyer, C., Leung, N., Waller, G., Perkins, S., Paice, N., \& Mitchell, J. (2005). Anger and bulimic psychopathology: Gender differences in a nonclinical group. International Journal of Eating Disorders, 37, 69-71.

Milligan, R. J., \& Waller, G. (2000). Anger and bulimic psychopathology among nonclinical women. International Journal of Eating Disorders, 28, 446-450.

Milligan, R. J., Waller, G., \& Andrews, B. (2002). Eating disturbances in female prisoners: The role of anger. Eating Behaviors, 3, 123-132.

Mond, J. M., Hay, P. J., Rodgers, B., Owen, C., \& Beumont, P. J. V. (2004). Validity of the eating disorder examination questionnaire (EDE-Q) in screening for eating disorders in community samples. Behaviour Research and Therapy, 42, 551-567.

Nolen-Hoeksema, S. (1991). Responses to depression and their effects on the duration of depressive episodes. Journal of Abnormal Psychology, 100, 569-582.

Schotte, D. E., \& Stunkard, A. J. (1987). Bulimia vs bulimic behaviours on a college campus. Journal of the American Medical Association, 258, 1213-1215.

Spielberger, C. D. (1996). State-Trait Anger Expression Inventory: Professional manual. Odessa, FL: PAR Psychological Assessment Resources, Inc.

Spielberger, C. D. (1999). Manual for the State-Trait Anger Expression Inventory-2. Odessa, FL: Psychological Assessment Resources.

Stice, E., Agras, W. S., Telch, C. F., Halmi, K. A., Mitchell, J. E., \& Wilson, T. (2001). Subtyping binge eatingdisordered women along dieting and negative affect dimensions. International Journal of Eating Disorders, 30, 11-27.

Timmerman, G. M. (1999). Binge Eating Scale: Further assessment of validity and reliability. Journal of Applied Biobehavioral Research, 4, 1-12.

Waller, G., Babbs, M., Milligan, R., Meyer, C., Ohanian, V., \& Leung, N. (2003). Anger and core beliefs in the eating disorders. International Journal of Eating Disorders, 34, 118-124.

Wilfley, D. E., Schwartz, M. B., Spurrell, E. B., \& Fairburn, C. G. (1997). Assessing the specific psychopathology of binge eating disorder patients: Interview or selfreport? Behavior Research and Therapy, 35, 11511159. 